\title{
Real-Time Emulation and Simulation System of Asynchronous Motor Consumption
}

\author{
Filipe Sousa, João Spínola, Nuno Moreira, Pedro Faria, Zita Vale \\ GECAD - Research Group on Intelligent Engineering and Computing for Advanced Innovation \\ and Development, Institute of Engineering, Polytechnic of Porto (ISEP/IPP), Portugal \\ \{ffeso, jafps, nnmor, pnfar, zav\}@isep.ipp.pt
}

\begin{abstract}
Electric power systems have been altered at the operating and planning level in the last years. Evidence of this was the liberalization of the energy market and the implementation of concepts such as smart grids and the active participation of consumers through demand response programs. The main objective of the present paper is to propose an emulation model for asynchronous motors, in the scope of dynamic measurement and control of loads, included in demand response programs. Since this type of electric motor is of upmost importance in the current consumption context, it was necessary to model consumption and evaluate its impact on the power system. The development of this paper aims to evaluate the system's capabilities using the motor as a load, and a wind emulator. Thus, we can represent various applications (e.g. division of a house) and realize the effect of distributed generation in the operation of the electric power system. Keywords: Load metering, Real-time simulation, Benchmarking, Emulator
\end{abstract}

\section{Introduction}

Electric Power Systems (EPS) have been the subject of major changes in the operating paradigm over the last decade. Nowadays, the increasing use of distributed generation instead of traditional large centralized production, is one of the main approaches these days. In addition to difficulties in maintenance, these large plants use primary fossil energy sources, such as coal and oil, which contribute largely to high pollutant emissions [1]. The dependency of fossil fuels to which the EPS was subject has boosted the commitment to renewable energy sources (RES) by several countries. This kind of initiative is very effective, since the use of fossil fuels presents many problems. The strong price volatility associated with the scarcity of resources from the buyer entity, together with the political and financial instability of the seller countries, represents relevant issues to the future implementation of fossil-based generation. As mentioned before, high pollutant emissions coupled with a growing environmental concern have boosted the focus on alternative energy sources, [2] and [3].

The present work was done and funded in the scope of the following project: NetEfficity Project (P2020 - 18015); and UID/EEA/00760/2013 funded by FEDER Funds through COMPETE program and by National Funds through FCT. 
The liberalization of the EPS has given rise to the concept of Smart Grids. This concept includes the activities of all connected users on the same system, such us, advanced information, network control and communication technology, making it possible to save energy and reduce consumption costs [4]. This concept is characterized by the integration of several complementary components and the cooperation of several subsystems, that coexist within the electrical grid. In [5], it's demonstrated the various components that are associated with smart grids. The great push of the Smart Grids is associated to the capacity of bidirectional communication between producer and final consumer, regulating the generation through Demand Response (DR), i.e. to design a real-time and cost-effective response, it is necessary that the consumers adjust to the amount of power that is delivered and behaviour. In this context, costly features as energy losses and spinning reserve can be avoided [6]. The use of strategies, which include price fluctuations or even incentive payments, seek to reduce energy consumption at higher prices on the wholesale market or when the reliability of the system is compromised [4], [7] and [8]. The DR includes all intentional changes in energy consumption, either by time of use, level of demand at critical times, or patterns of consumption adjusted to market prices, [4], [7], [9] and [10]. Since RES are not constant, its use does not guarantee a stable operation. New methodologies require a careful evaluation of, the developed software and its applicability. In [11], one can see the relations between the ambient and application conditions that can be applied in system's evaluation, [11] and [12]. As there is no perfect model solution for DR, computational simulation or emulation arise as the least costly alternatives. In sum, there is the need for a platform that creates a reasonable compromise between simulation and emulation, combining software and real-life applications.

The proposed emulation method intends to provide a model for the simulation of load profiles, with a real-time communication infrastructure of monitoring and control that enables its user a diverse range of operation scenarios. The paper is divided into five sections. Section 2 details the importance of multi-agent systems (MAS) in this approach, while section 3 specifies the components of the emulation method. In Section 4 , it is presented the case study and its results for the application of emulation method, and as for Section 5 , it is shown the conclusions of the results obtained.

\section{Multi-Agent Systems}

The concept of MAS is very useful in the context of smart grids, since it defines autonomous, local, and distributed managers of systems, that are connected to form an agent network of cooperation and interaction that simplifies processes [13]. In this way, MAS (intelligent or not) can be integrated as a solution for monitoring and controlling distributed systems. MAS are often applied in energy markets simulation, namely, in terms of energy bids and the interaction between several entities, as mentioned in [14] and [15]. Per example, MASCEM, [16] and [17], represents a multi-agent tool that can model several electricity markets and their operation, considering agent's interaction, their characteristics, behaviours and interests, [15] and [17].

In [18], the authors present a solution for the practical implementation of MAS, SOICAM, and how these can be integrated with a scheduling platform, MASGriP. In 
this way, this kind of approach can enable interactions between several agents in a simulated environment, that can accurately demonstrate the usefulness of these systems in real applications, such as, residential energy managers, sensor hubs, operators, aggregators, amongst others. In the present paper, it is assumed the existence of two agents, namely, the user of the software (computer) and the monitoring equipment (master analyser), communicating through TCP/IP protocol. The interaction between these two enables a multi-agent system, related to the implementation of smart grid measures.

\section{Developed Solution}

This section presents the communications and electrical connections that compose the implemented platform.

\subsection{Equipment}

- OPAL. is a real-time simulator, allowing several simulation and control systems, including hardware-in-the-loop (HIL) testing, rapid prototype control, complex power networks, distributed energy resources and electronic power systems. The simulation can be in real time, i.e. in a time as low as $10 \mu$ s [18].

- PLC. is a control device, which executes a program that monitors inputs and handles the outputs. From a computer, it is possible to communicate with the PLC and control equipment, in addition to being able to receive data as well. They are also distinguished by their reliability in a harsh environment, [19] and [20].

- DIGIWATT. a digital multimeter with floating inputs for voltage, current and electrical power, that can assimilate different states of all the equipment which it is connected. The DIGIWATT is capable of performing power measurement considering the DC component, a single phase, and the three-phase balance.

- GRANMECA. an equipment for exposing three mechanical values: torque, speed and mechanical power. It makes a reading of the speed and torque through the voltage received from the tachymetric dynamo and the torque sensor, respectively. The equipment has an adjustable knob that controls the magnetic brake.

- VISIREAL. Enables analogue measurement outputs (GRANMECA and DIGIWATT) to record and display electrical and mechanical quantities on a computer. The LOGIREAL software, which works together with VISIREAL, allows the user to view the variables in real time, through an RS-232 connection that links the measuring device to a computer. During real time acquisition, the several parameters are shown through curves or in numerical displays.

- ANALYSERS. Architecture based in only one master analyser (Janitza ${ }^{\circledR}$ UMG 604) connected to motor A, being motors B and C considered slaves (Janitza ${ }^{\circledR}$ UMG 103), that are more limited but adequate and less costly.

\subsection{Communication scheme}

The developed solution is represented in Fig. 1. This is divided into three distinct phases: decision, monitoring and control, and finally, simulation/actuation. 
The decision phase allows the user to access the data coming from the installation and define preferences. This access is versatile since it can be done locally through a computer with network access (via wireless or network cable), or through remote access using a VPN connection. The OPAL, PLC and energy analysers are also connected to the network to be accessible to the user.

In the monitoring and control phase, it is important to emphasize the communication with the energy analyser, since it will allow requests to be made, and after answers received. The communication protocol used with the energy analyser is Modbus TCP/IP (Transmission Control Protocol / Internet Protocol), two independent protocols but working together. Modbus is a communication protocol that has the benefits of flexibility and easy implementation. It can be used for communication between computers and microcontrollers, PLCs and other devices. In the present paper, operation was only considered through the PLC, and OPAL is not being used.

In the simulation/actuation, the communication network uses the master device controlled by the computer, to establish a connection with the slaves. The analyser waits for a connection request from the computer and, when this is established the analyser responds to the requests from the computer until it closes the connection.

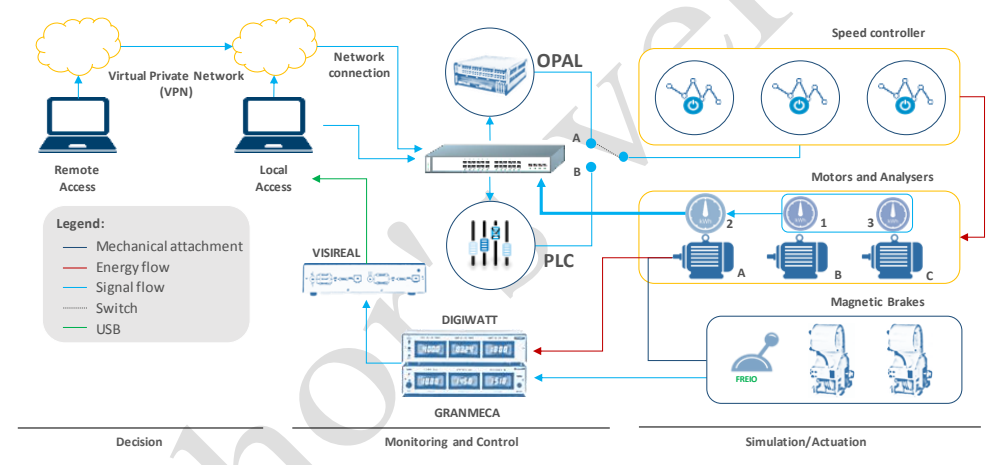

Fig. 1. Communication scheme.

It is important to note that the installation is equipped with three position switches, to make the control option to be used: choice is made between OPAL, PLC or isolated. It is also possible to vary the brake intensity, i.e. the torque. The electrical and mechanical quantities obtained in both equipment are transmitted to a computer where the analysis software is installed. In addition to obtaining information through the energy analyser via MATLAB®, one can also get information and command from the PLC and OPAL. In the third phase, the study considers 3 motors: A $(3000 \mathrm{~W}), \mathrm{B}(3000 \mathrm{~W})$, and $\mathrm{C}(1500 \mathrm{~W})$ with one analyser each, of which the master is analyser 2 (motor A). This means that analyser 2 , in addition to collecting data from the motor to which is connected, also receives data from the other two analysers, 1 (motor B) and 3 (motor C).

\subsection{Wiring scheme}

The wiring diagram used in the installation is presented in Fig. 2. All motors are starcoupled and powered by a three-phase source, however some equipment has single- 
phase power, namely the metering and brake devices. Motor A is coupled to a magnetic brake, a torque sensor, and a tachymetric dynamo. The magnetic brake serves to regulate the torque that the motor is developing, and it can be adjusted through a potentiometer. The reading obtained from the torque sensor is fundamental for the user to regulate the brake, since in a model environment this is one of the parameters that can be modified in this approach. As for the tachymetric dynamo, it allows the measurement of the motor's speed, through an analogue connection.

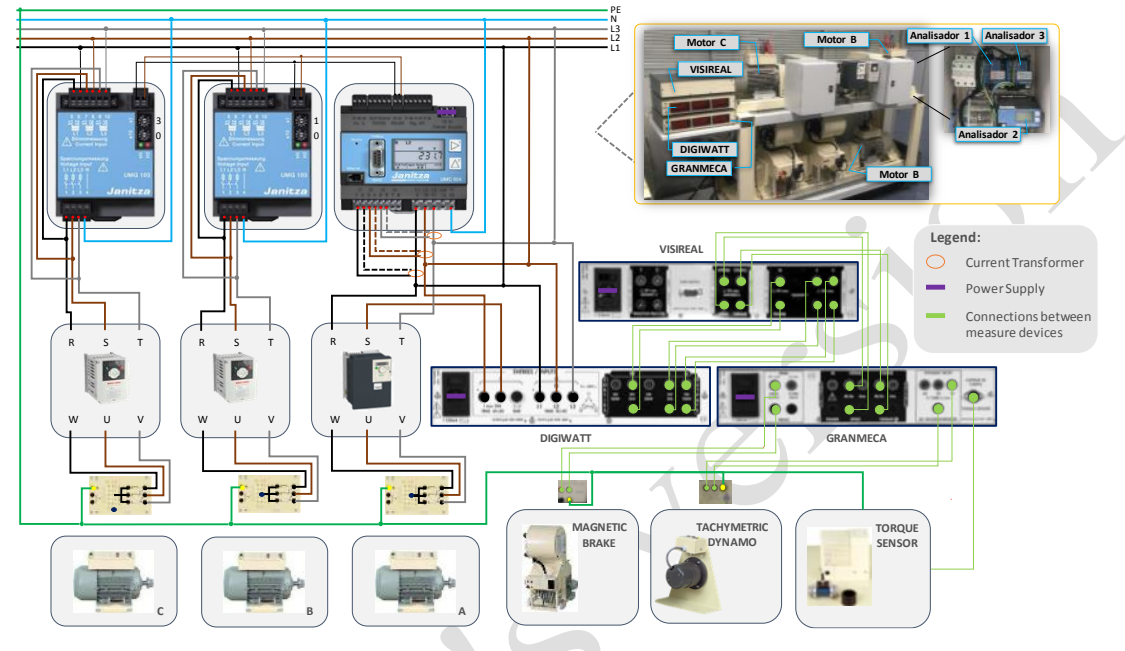

Fig. 2. Wiring scheme.

In short, regarding motor $\mathrm{A}$, it is coupled to a magnetic brake, tachymetric dynamo, and a torque sensor, while motors $\mathrm{B}$ and $\mathrm{C}$, are only measured by the analysers. The three-phase source is connected to the variable speed drive, however, firstly, connecting to the energy analyser allowing for voltage and current measurements. In this way, one has a realistic measurement that includes the consumption of the variable speed drive, which otherwise, the measured load would be incorrect since it does not include all load components. Each analyser has an address, which is a necessary information for identification of the analysers in the network, being for motor B the address 1, and for motor $\mathrm{C}$, the address 3 . An algorithm is necessary to obtain the electrical quantities with user defined sampling time and desired parameters. Thus, the communication is established through the master analyser allowing direct communication with the user, which is connected to the local network. Three versions of the algorithm were developed (Table 1).

- Version 1 - collects measurements of the master analyser and the two slave analysers, plotting data after emulation is finalized.

- Version 2 - collects data only from the master analyser, plotting data after emulation.

- Version 3 - collects data only from the master analyser, plotting data in real-time.

The user's communication with the analyser was established through a code developed in MATLAB®. Only the master analyser is capable of being connected to the network, thus, it is necessary to use RS-485 communication protocol to obtain the information collected by the other analysers. The master device is identified by unitID 
that in this case corresponds to " 02 ", while the analyser for motor 2 and 3 , by " 01 " and "03", respectively. Considering what was mentioned before, this paper aims to simulate operation scenarios of energy systems in a practical environment.

Table 1. Characteristics of the different communication algorithm's versions.

\begin{tabular}{|l|c|c|c|}
\cline { 2 - 4 } \multicolumn{1}{c|}{} & Version 1 & Version 2 & Version 3 \\
\hline \hline Number of analysers & 3 & \multicolumn{2}{c|}{1} \\
\hline Total simulation time $\left(\mathrm{t}_{\mathrm{s}}\right)$ & \multicolumn{3}{|c|}{ Defined by user } \\
\hline Communication time $\left(\mathrm{t}_{\mathrm{c}}\right)$ & $0.9 \mathrm{~s}$ & $0.2 \mathrm{~s}$ & $0.05 \mathrm{~s}$ \\
\hline Sampling time & $\mathrm{t}_{\mathrm{c}}$ & $\mathrm{t}_{\mathrm{c}}$ & $\mathrm{T}_{\text {iter }}$ \\
\hline Each iteration time $\left(\mathrm{T}_{\text {iter }}\right)$ & $\mathrm{t}_{\mathrm{c}}$ & $\mathrm{t}_{\mathrm{c}}$ & $\mathrm{t}_{\mathrm{c}}+\mathrm{t}_{\mathrm{t}}+\mathrm{t}_{\mathrm{g}}$ \\
\hline Real-Time Graphing & No & No & Yes \\
\hline Data processing each iteration & No & No & Yes \\
\hline
\end{tabular}

Note: the presented times are approximate and may vary on each iteration.

The objectives relate to equipment installation in GECAD laboratory, namely:

- Development of an algorithm in MATLAB® with Ethernet connection to the analyser, through Modbus TCP / IP communication protocol;

- Evaluation of sampling time, i.e. time interval between requests to the analyser;

- Analysis of the processing times required for the extraction and visualization of results, considering the effect of their veracity.

- Evaluation of the potential of the system using the motor as a load, allowing it to represent several applications (e.g. lighting system, among others);

- Integration of a wind emulator, to portray the inclusion of distributed production in an energy system, and its effect on the operation of the same.

\section{$4 \quad$ Case Study \& Results}

In addition to the measurements of motor $\mathrm{A}$, the active power of the wind emulator is also shown, which is used to simulate energy production from a wind turbine. The load (motor), wind emulator and power grid, are the three resources involved in this study. Three scenarios are considered: in scenario A, it is represented the motor's operation when there is no energy production, and therefore all the load is satisfied by the power grid; in scenario $\mathrm{B}$, wind production is higher than the motor consumption, and thus, can feed the load and inject power in the grid; scenario $\mathrm{C}$ considers that the motor's consumption is partially higher than the available wind generation, being needed some contribution from the main network. Fig. 3 shows the motor's electrical magnitudes, the production's active power, and the energy flow over time, in the 3 scenarios.

By analysing the energy flows, it is possible to notice that the simulation starts without production and, therefore, the load is fed by the electric grid. Around $5 \mathrm{~s}$ we started to have production, but still insufficient to feed the load. At $25 \mathrm{~s}$ the output is greater than what is required for consumption and, therefore, the wind turbine feeds the load and injects power into the main network. At 50 seconds, there are large variations in the production versus consumption balance almost instantly changing the energy flow, stabilizing when wind production is insufficient to meet consumption. After this and at the end of the simulation, the consumption is supported again by the energy produced 
by the wind turbine. The characteristics of the wind emulator are: Max. current - $5 \mathrm{~A}$; Nominal power - $1.2 \mathrm{kVA}$; Speed - $1800 \mathrm{rpm}$.

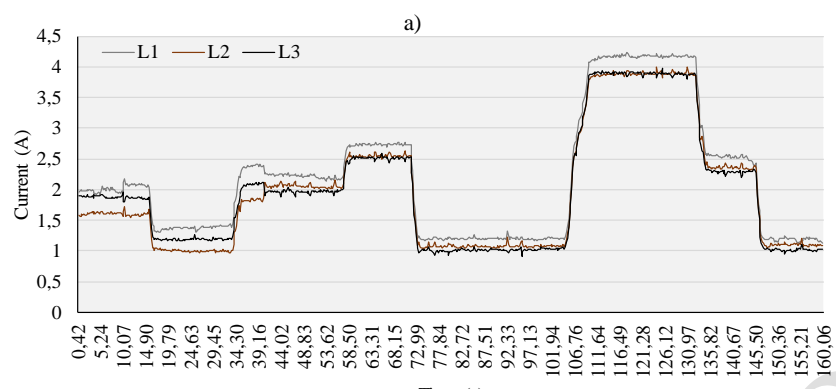

Time (s)

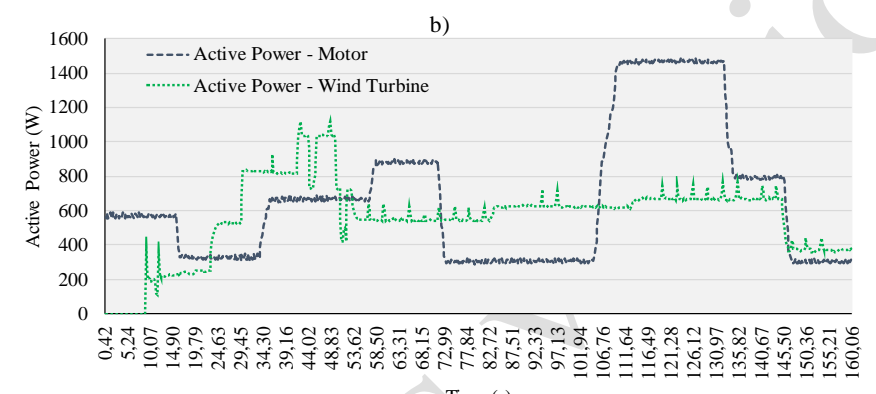

Time (s)

Fig. 3. Graphs of the motor A with wind power production. A) Efficient currents in Motor A; B) Active Power in Motor A and in the wind turbine.

\section{Conclusions}

The smart grids concept was one of the great innovations, since new concepts such as load control and DR emerge has solutions to a better energy management. This work aimed to evaluate the impact of electric loads, namely asynchronous motors in the context of DR programs, to improve decision support and operation awareness. By implementing an emulation framework, it is possible to simulate operation scenarios that imply different analysis and decisions based on the load profile. After analysing all the case studies, it is concluded that each situation requires a different evaluation of what algorithm to be used, and in what context it is applied. From the analysis made, version 1 considers requests for the 3 analysers, and thus is slower than the other versions. In the other versions, 2 compared to 3 presents a great advantage of reduced sampling time, which allows a greater number of measurements, however, version 2 does not allow the visualization of the measurements in real time, capability that version 3 has.

\section{References}

1. H. Gabriel and V. Morais, "Gestão de Recursos Energéticos nas SmartGrids," 2010. 
2. International Energy Agency, "Resources to Reserve 2013," New Dir. Youth Dev., vol. 2013, no. 140, pp. 5-8, 2013.

3. IEA, OECD, and World Bank Group, "The Scope of Fossil Fuel Subsidies in 2009 and a Roadmap for Phasing Out Fossil Fuel Subsidies,” Jt. Rep. Prep. G20 Summit Seoul (South Korea), 11-12 Nov., 2010.

4. S. Ali, R. Ahmad, and D. Kim, "A study of pricing policy for demand response of home appliances in smart grid based on M2M," Proc. - 10th Int. Conf. Front. Inf. Technol. FIT 2012, pp. 231-236, 2012.

5. K. De Craemer and G. Deconinck, "Analysis of State-of-the-art Smart Metering Communication Standards," Proc. 5th Young Res. Symp., pp. 1-6, 2010.

6. S. Ghosh, X. A. Sun, and X. Zhang, "Consumer profiling for demand response programs in smart grids," 2012 IEEE Innov. Smart Grid Technol. - Asia, ISGT Asia 2012, pp. 1-6, 2012.

7. S. H. Li, D. Zhang, B. Roget, and Z. O'Neill, "Integrating Home Energy Simulation and Dynamic Electricity Price for Demand Response Study," IEEE Trans. Smart Grid, vol.5, no.2, pp. 779-788, 2014.

8. P. Faria, Z. Vale, and J. Baptista, "Demand response programs design and use considering intensive penetration of distributed generation," Energies, vol. 8, no. 6, pp. 6230-6246, 2015.

9. P. Faria, "Demand Response in future power systems management-A conceptual framework and simulation tool," 2011.

10. S. M. Hakimi and S. M. Moghaddas-Tafreshi, "Optimal Planning of a Smart Microgrid Including Demand Response and Intermittent Renewable Energy Resources,” vol. 5, no. 6, pp. 2889-2900, 2014.

11. J. Gustedt, E. Jeannot, and M. Quinson, "Experimental Methodologies for Large-Scale Systems : a Survey," 2009.

12. Y. H. Wang and I. C. Wu, "Achieving high and consistent rendering performance of java AWT/Swing on multiple platforms," Softw. - Pract. Exp., vol. 39, no. 7, pp. 701-736, 2009.

13. Qin Li, Graeme Smith, Formal development of multi-agent systems using MAZE, Science of Computer Programming, Volume 131, 1 December 2016, Pages 126-150, ISSN 0167-6423, http://dx.doi.org/10.1016/j.scico.2016.04.008.

14. Tiago Pinto, João Barreto, Isabel Praça, Tiago M. Sousa, Zita Vale, E.J. Solteiro Pires, Six thinking hats: A novel metalearner for intelligent decision support in electricity markets, Decision Support Systems, Volume 79, November 2015, Pages 1-11, ISSN 0167-9236, http://dx.doi.org/10.1016/j.dss.2015.07.011.

15. Francisco Silva, Brígida Teixeira, Tiago Pinto, Gabriel Santos, Zita Vale, Isabel Praça, Generation of realistic scenarios for multi-agent simulation of electricity markets, Energy, Volume 116, Part 1, 1 December 2016, Pages 128-139, ISSN 0360-5442, http://dx.doi.org/10.1016/j.energy.2016.09.096.

16. Gabriel Santos, Tiago Pinto, Hugo Morais, Tiago M. Sousa, Ivo F. Pereira, Ricardo Fernandes, Isabel Praça, Zita Vale, Multi-agent simulation of competitive electricity markets: Autonomous systems cooperation for European market modeling, Energy Conversion and Management, Volume 99, 15 July 2015, Pages 387-399, ISSN 0196-8904, http://dx.doi.org/10.1016/j.enconman.2015.04.042.

17. Gabriel Santos, Tiago Pinto, Isabel Praça, Zita Vale, MASCEM: Optimizing the performance of a multiagent system, Energy, Volume 111, 15 September 2016, Pages 513-524, ISSN 0360-5442, http://dx.doi.org/10.1016/j.energy.2016.05.127.

18. L. Gomes, F. Fernandes, P. Faria, M. Silva, Z. Vale and C. Ramos, "Contextual and environmental awareness laboratory for energy consumption management," 2015 Clemson University Power Systems Conference (PSC), Clemson, SC, 2015, pp. 1-6., doi: 10.1109/PSC.2015.7101678

19. OPAL-RT TECHNOLOGIES, "Introducing the OP5600 Family, OPAL-RT's Real-Time Digital Simulators," 2016. [Online]. Available: http://www.opal-rt.com/product/op5600-hil-hardware-in-theloop-computer-and-IO-system. [Accessed: 05-Sep-2016].

20. ISA, "Programmable logic controllers: Hardware, software architecture." [Online]. Available: https://www.isa.org/standards-publications/isa-publications/intechmagazine/2010/december/automation-basics-programmable-logic-controllers-hardware-softwarearchitecture/. [Accessed: 20-Sep-2016]. 